\title{
Unifying Parallels
}

\author{
Claire Gardent \\ Computational Linguistics \\ University of the Saarland \\ Saarbrücken, Germany \\ claire@coli.uni-sb.de
}

\begin{abstract}
I show that the equational treatment of ellipsis proposed in (Dalrymple et al., 1991) can further be viewed as modeling the effect of parallelism on semantic interpretation. I illustrate this claim by showing that the account straightforwardly extends to a general treatment of sloppy identity on the one hand, and to deaccented foci on the other. I also briefly discuss the results obtained in a prototype implementation.
\end{abstract}

\section{Introduction}

(Dalrymple et al., 1991; Shieber et al., 1996) (henceforth DSP) present a treatment of VPellipsis which can be sketched as follows. An elliptical construction involves two phrases (usually clauses) which are in some sense structurally parallel. Whereas the first clause (we refer to it as the source) is semantically complete, the second (or target) clause is missing semantic material which can be recovered from the source.

Formally the analysis consists of two components: the representation of the overall discourse (i.e. source and target clauses) and an equation which permits recovering the missing semantics.

\begin{tabular}{|l|l|}
\hline Representation & $S \wedge R\left(T_{1}, \ldots, T_{n}\right)$ \\
\hline Equation & $R\left(S_{1}, \ldots, S_{n}\right)=S$ \\
\hline
\end{tabular}

$S$ is the semantic representation of the source, $S_{1}, \ldots, S_{n}$ and $T_{1}, \ldots, T_{n}$ are the semantic representations of the parallel elements in the source and target respectively and $R$ represents the relation to be recovered. The equation is solved using Higher-Order Unification (HOU): Given any solvable equation $M=N$, HOU yields a substitution of terms for free variables that makes $M$ and $N$ equal in the theory of $\alpha \beta \eta$-identity.

The following example illustrates the workings of this analysis:

$$
\text { Jon likes Sarah and Peter does too. }
$$

In this case the semantic representation and the equation associated with the overall discourse are:

\begin{tabular}{|l|l|}
\hline Representation & $\operatorname{like}(j, s) \wedge R(p)$ \\
\hline Equation & $R(j)=\operatorname{like}(j, s)$ \\
\hline
\end{tabular}

For this equation, HOU yields the substitution ${ }^{1}$ :

$$
\{R \leftarrow \lambda x . \operatorname{like}(x, s)\}
$$

and as a result, the resolved semantics of the target is:

$$
\lambda x . \operatorname{like}(x, s)(p) \equiv \operatorname{like}(p, s)
$$

The DSP approach has become very influential in computational linguistics for two main reasons. First, it accounts for a wide range of observations concerning the interaction of VPellipsis, quantification and anaphora. Second, it bases semantic construction on a tool, HOU, which is both theoretically and computationally attractive. Theoretically, HOU is well-defined and well-understood - this permits a clear understanding of both the limitations and the predictions of the approach. Computationally, it has both a declarative and a procedural interpretation - this supports both transparency and implementation.

\footnotetext{
${ }^{1}$ As (Dalrymple et al., 1991) themselves observe, HOU also yields other, linguistically invalid, solutions. For a proposal on how to solve this over-generation problem, see (Gardent and Kohlhase, 1996b; Gardent et al., 1999).
} 
In this paper, I start (section 2) by clarifying the relationship between DSP's proposal and the semantic representation of discourse anaphors. In section 3 and 4 , I then show that the HOU-treatment of ellipsis naturally extends to provide:

- A treatment of the interaction between parallelism and focus and

- A general account of sloppy identity

Section 6 concludes and compares the approach with related work.

\section{Representing discourse anaphors}

The main tenet of the DSP approach is that interpreting an elliptical clause involves recovering a relation from the source clause and applying it to the target elements. This leaves open the question of how this procedure relates to sentence level semantic construction and in particular to the semantic representation of VPellipsis. Consider for instance the following example:

Jon runs but Peter doesn't.

Under the DSP analysis, the unresolved semantics of (2) is (3)a and equation (3) b is set up. HOU yields the solution given in (3)c and as a result, the semantics of the target clause Peter doesn't is (3)d.
a. $\operatorname{pos}(\operatorname{run}($ jon $)) \wedge R($ neg $)($ peter $)$
b. $R($ pos $)(j o n)=\operatorname{pos}(\operatorname{run}($ jon $))$
c. $\quad\{R \rightarrow \lambda O \lambda x . O(\operatorname{run}(x))\}$
d. $\lambda O \lambda x . O($ run $(x))($ neg $)($ peter $)$ $n e g($ run(peter $))$

It is unclear how the semantic representation (3)a comes about. Under a Montague-type approach where syntactic categories map onto semantic types, the semantic type of a VPEllipsis is $(e t)$, the type of properties of individuals i.e. unary relations, not binary ones. And under a standard treatment of subject NPs and auxiliaries, one would expect the representation of the target clause to be neg $(P($ peter $))$ not $P($ neg $)$ (peter $)$. There is thus a discrepancy between the representation DSP posit for the target, and the semantics generated by a standard, Montague-style semantic construction module.
Furthermore, although DSP only apply their analysis to VP-ellipsis, they have in mind a much broader range of applications:

[...] many other elliptical phenomena and related phenomena subject to multiple readings akin to the strict and sloppy readings discussed here may be analysed using the same techniques (Dalrymple et al., 1991, page 450).

In particular, one would expect the HOUanalysis to support a general theory of sloppy identity. For instance, one would expect it to account for the sloppy interpretation (I'll kiss you if you don't want me to kiss you) of (4).

$$
\begin{aligned}
& \text { I'll [help you] } \left.]^{1} \text { if you [want me to }\right]^{2} \text {. } \\
& \text { I'll kiss you if you don't } t_{2} \text {. }
\end{aligned}
$$

But for such cases, the discrepancy between the semantic representation generated by semantic construction and the DSP representation of the target is even more obvious. Assuming help and kiss are the parallel elements, the equation generated by the DSP proposal is:

$$
R(h)=w t(\text { you }, h(i, y o u)) \rightarrow h(i, y o u)
$$

and accordingly, the semantic representation of the target is $\neg R(k)$ which is in stark contrast with what one could reasonably expect from a standard semantic construction process namely: $\neg P($ you $) \rightarrow k(i$, you $)$.

What is missing is a constraint which states that the representation of the target must unify with the semantic representation generated by the semantic construction component. If we integrate this constraint into the DSP account, we get the following representations and constraints:

\begin{tabular}{|l|l|}
\hline Representation & $S \wedge R\left(T_{1}, \ldots, T_{n}\right)$ \\
\hline Equations & $R\left(S_{1}, \ldots, S_{n}\right)=S$ \\
& $R\left(T_{1}, \ldots, T_{n}\right)=T$ \\
\hline
\end{tabular}

where $T$ is the semantic representation generated for the target by the semantic construction module. The second equation requires that this representation $T$ unifies with the representation of the target postulated by DSP.

With this clarification in mind, example (2) is handled as follows. The semantic representation 
of (2) is (6)a where the semantic representation of the target clause is the representation one would expect from a standard Montague-style semantic construction process. The equations are as given in (6)b-c where $C$ represents the semantics shared by the parallel structures and $P$ the VP-Ellipsis. HOU then yields the solution in (6)d: the value of $C$ is that relation shared by the two structures i.e. a binary relation as in DSP. However the value of $P$ (the semantic representation of the VPE) is a property - as befits a verbal phrase.

$$
\begin{array}{ll}
\text { a. } & \operatorname{pos}(\operatorname{run}(\text { jon })) \wedge \operatorname{neg}(P(\text { peter })) \\
\text { b. } & C(\text { pos })(\text { jon })=\operatorname{pos}(\text { run }(\text { jon })) \\
\text { c. } & C(\text { neg })(\text { peter })=\operatorname{neg}(P(\text { peter })) \\
\text { d. } & \{C \rightarrow \lambda O \lambda x \cdot O(\text { run }(x)), P \rightarrow \\
& \lambda x \cdot \operatorname{run}(x)\} \\
\text { e. } & \lambda O \lambda x O(\text { run }(x))(\text { neg })(\text { peter }) \quad \equiv \\
& n e g(\operatorname{run}(\text { peter }))
\end{array}
$$

In sum, provided one equation is added to the DSP system, "the relation between the $\mathrm{HOU}$-approach to $\mathrm{VP}$-ellipsis and standard Montague-style semantic construction becomes transparent. Furthermore it also becomes immediately obvious that the DSP approach does indeed generalise to a much wider range of data than just VP-Ellipsis. The key point is that there is now not just one, but several, free variables coming into play; and that although the free variable $C$ always represents the semantics shared by two parallel structures, the free variable(s) occuring in the semantic representation of the target may represent any kind of unresolved discourse anaphors - not just ellipsis. Consider the following example for instance:

$J o n^{1}$ took his 1 wife to the station. No, BILL took his wife to the station.

There is no ellipsis in the target, yet the discourse is ambiguous between a strict and a sloppy interpretation ${ }^{2}$ and one would expect the HOU-analysis to extend to such cases. Which indeed is the case. The analysis goes as follows.

\footnotetext{
${ }^{2}$ I assume that in the target took his wife to the station is deaccented. In such cases, it is clear that the ambiguity of his is restricted by parallelism i.e. is a sloppy/strict ambiguity rather than just an ambiguity in the choice of antecedent.
}

As for ellipsis, anaphors in the source are resolved, whereas discourse anaphors in the target are represented using free variables (alternatively, we could resolve them first and let HOU filter unsuitable resolutions out). Specifically, the target pronoun his is represented by the free variable $X$ and therefore we have the following representation and equations:

\begin{tabular}{|l|l|}
\hline Representation & $\begin{array}{l}t k(j, \text { wife_of }(j), s) \\
\wedge t k(b, \text { wife_of }(X), s)\end{array}$ \\
\hline Equations & $C(j)=t k(j$, wife_of $(j), s)$ \\
& $C(b)=t k(b$, wife_of $(X), s)$ \\
\hline
\end{tabular}

HOU yields inter alia two solutions for these equations, the first yielding a strict and the second, a sloppy reading:

$$
\begin{aligned}
& \{C \leftarrow \lambda z \cdot t k(z, \text { wife-of }(j), s), X \leftarrow j\} \\
& \{C \leftarrow \lambda z . t k(z, \text { wife_of }(z), s), X \leftarrow b\}
\end{aligned}
$$

Thus the HOU-approach captures cases of sloppy identity which do not involve ellipsis. More generally, the HOU-approach can be viewed as modeling the effect of parallelism on interpretation. In what follows, I substantiate this claim by considering two such cases: first, the interaction of parallelism and sloppy identity and second, the interaction of parallelism and focus.

\section{Parallelism and Focus}

Since (Jackendoff, 1972), it is widely agreed that focus can affect the truth-conditions of a sentence ${ }^{3}$. The following examples illustrate this, where upper-letters indicate prosodic prominence and thereby focus.

$$
\begin{aligned}
& \text { a. Jon only introduced MARY to Sue. } \\
& \text { b. Jon only introduced Mary to SUE. }
\end{aligned}
$$

Whereas (8a) says that the only person introduced by Jon to Sue is Mary, ( $8 \mathrm{~b})$ states that the only person Jon introduced Mary to, is Sue.

To capture this effect of focus on semantics, a focus value $e^{4}$ is used which in essence, is the

\footnotetext{
${ }^{3}$ The term focus has been put to many different uses. Here I follow (Jackendoff, 1972) and use it to refer to the semantics of that part of the sentence which is (or contains an element that is) prosodically prominent.

${ }^{4} \mathrm{This}$ focus value is defined and termed differently by different authors: Jackendoff (Jackendoff, 1972) calls it the presuppositional set, Rooth (Rooth, 1992b) the Alternative Set and Krifka (Krifka, 1992) the Ground.
} 
set of semantic objects obtained by making an appropriate substitution in the focus position. For instance, in (Gardent and Kohlhase, 1996a), the focus value of $(8 \mathrm{a})$ is defined with the help of the equation:

$$
\begin{array}{|l|l|}
\hline \text { Focus Value Equation } & S e m=X(F) \\
\hline
\end{array}
$$

where $S e m$ is the semantic of the sentence without the focus operator (e.g. intro $(j, m, s)$ for (8)), $F$ represents the focus and $X$ helps determine the value of the focus variable (written $\bar{X}$ ) as follows:

Definition 3.1 (Focus value) Let $X=\lambda x . \phi$ be the value defined by the focus value equation and $\tau$ be the type of $x$, then the Focus value derivable from $X$, written $\bar{X}$, is $\{\phi \mid$ $\left.x \in w f_{\tau}\right\}$.

Given (8a), the focus value equation is thus (9a) with solution (9b); the focus value derived from it is $(9 c)$ and the semantics of $(8 a)$ is $(9 d)$ which given $(9 \mathrm{c})$ is equivalent to $(9 \mathrm{e})$.

$$
\begin{array}{ll}
\text { a. } & \text { intro }(j, m, s)=X(m) \\
\text { b. } & \{X \leftarrow \lambda x . i n t r o(j, x, s)\} \\
\text { c. } & \bar{X}=\left\{\text { intro }(j, x, s) \mid x \in w f_{e}\right\} \\
\text { d. } & \forall P[P \in X \wedge P \rightarrow P=\text { intro }(j, m, s)] \\
\text { e. } & \forall P\left[P \in\left\{\text { intro }(j, x, s) \mid x \in w f_{e}\right\} \wedge\right. \\
& P \rightarrow P=\operatorname{intro}(j, m, s)]
\end{array}
$$

In English: the only proposition of the form John introduced $x$ to Sue that is true is the proposition John introduced Mary to Sue.

Now consider the following example:

$$
\begin{aligned}
& \text { a. Jon only likes MARY } \\
& \text { b. No, PETER only likes Mary. }
\end{aligned}
$$

In a deaccenting context, the focus might be part of the deaccented material and therefore not prosodically prominent. Thus in (10)b, the semantic focus Mary is deaccented because of the partial repetition of the previous utterance. Because they all use focus to determine the focus value and thereby the semantics of sentences such as (8a), focus deaccenting is a challenge for most theories of focus. So for instance, in the HOU-analysis of both (Pulman, 1997) and (Gardent and Kohlhase, 1996a), the right-hand side of the focus equation for (10b) becomes $F V(F)$ where neither $F V$ (the focus value) nor $F$ (the focus) are known. As a result, the equation is untyped and cannot be solved by Huet's algorithm (Huet, 1976).

The solution is simple: if there is no focus, there is no focus equation. After all, it is the presence of a focus which triggers the formation of a focus value.

But how do we determine the interpretation of (10b)? Without focus equation, the focus value remains unspecified and the representation of $(10 b)$ is:

$$
\forall P[P \in F V \wedge P \rightarrow P=\operatorname{like}(p, m)]
$$

which is underspecified with respect to $F V$.

(Rooth, 1992a) convincingly argues that deaccenting and VP-ellipsis are constrained by the same semantic redundancy constraint (and that VP-ellipsis is additionally subject to a syntactic constraint on the reconstructed VP). Moreover, (Gardent, 1999) shows that the equational constraints defined in (5) adequately characterise the redundancy constraint which holds for both VPE and deaccenting. Now example (10b) clearly is a case of deaccenting: because it repeats the VP of (10a), the VP only likes mary in (10b) is deaccented. Hence the redundancy constraint holding for both VPE and deaccenting and encoded in (5) applies ${ }^{5}$ :

$$
\begin{aligned}
C(j) & =\forall P[P \in\{\operatorname{like}(j, x)\} \wedge P \\
& \rightarrow P=\operatorname{like}(j, m)] \\
C(p) & =\forall P[P \in F V \wedge P \rightarrow P=\operatorname{like}(p, m)]
\end{aligned}
$$

These equations are solved by the following substitution:

$$
\begin{aligned}
\{C \leftarrow \quad & \lambda z \cdot \forall P[P \in\{\operatorname{like}(z, x)\} \wedge P \\
& \rightarrow P=\operatorname{like}(z, m)], \\
F V \leftarrow & \{\operatorname{like}(p, x)\}\}
\end{aligned}
$$

so that the interpretation of $(10 \mathrm{~b})$ is correctly fixed to:

$$
\forall P[P \in\{\operatorname{like}(p, x)\} \wedge P \rightarrow P=\operatorname{like}(p, m)]
$$

Thus, the HOU approach to deaccenting makes appropriate predictions about the interpretation of "second occurrence expressions"

\footnotetext{
${ }^{5}$ For lack of space, I shorten $\{l i k e(j, x) \mid x \in w f f\}$ to $\{\operatorname{like}(j, x)\}$
} 
$(\mathrm{SOE})^{6}$ such as (10b). It predicts that for these cases, the focus value of the source is inherited by the target through unification. Intuitively, a sort of "parallelism constraint" is at work which equates the interpretation of the repeated material in an SOE with that of its source counterpart.

Such an approach is in line with (Krifka, 1992) which argues that the repeated material in an SOE is an anaphor resolving to its source counterpart. It is also partially in line with Rooth's account in that it similarly posits an initially underspecified semantics for the target; It is more specific than Rooth's however, as it lifts this underspecification by unification. The difference is best illustrated by an example:

$$
\begin{aligned}
& \text { ?? Jon only likes SARAH. No, PETER } \\
& \text { only likes Mary. }
\end{aligned}
$$

Provided only likes Mary is deaccented, thị discourse is ill-formed (unless the second speaker knows Sarah and Mary to denote the same individual). Under the HOU-analysis this falls out of the fact that the redundancy constraint cannot be satisfied as there is no unifying substitution for the following equations:

$$
\begin{aligned}
C(j) & =\forall P[P \in\{\operatorname{like}(j, x)\} \wedge P \\
& \rightarrow P=\operatorname{like}(j, s)] \\
C(p) & =\forall P[P \in F V \wedge P \rightarrow P=\operatorname{like}(p, m)]
\end{aligned}
$$

In constrast, Rooth's approach does not capture the ill-formedness of (11) as it places no constraint on the interpretation of PETER only likes Mary other than that given by the compositional semantics of the sentence namely:

$$
\forall P[P \in F V \wedge P \rightarrow P=\operatorname{like}(p, m)]
$$

where $F V$ represents the quantification domain of only and is pragmatically determined. Without going into the details of Rooth's treatment of focus, let it suffice to say, that the first clause does actually provide the appropriate antecedent for this pragmatic anaphor so that despite its ill-formedness, (11) is assigned a fullfledged interpretation.

\footnotetext{
${ }^{6}$ The terminology is borrowed from (Krifka, 1995) and refers to expressions which partially or totally repeat a previous expression.
}

Nonetheless there are cases where pragmatic liberalism is necessary. Thus consider Rooth's notorious example:

\section{People who GROW rice usually only EAT rice}

This is understood to mean that people who grow rice usually eat nothing else than rice. But as the focus (RICE) and focus value $(\lambda x . e a t(p w g r, x))$ that need to be inherited by the target VP only EAT rice are simply not available from the previous context, the redundancy constraint on deaccenting fails to predict this and hence, fails to further specify the underspecified meaning of (12). A related case in point is:

We are supposed to TAKE maths and semantics, but I only LIKE semantics.

Again the focus on $L I K E$ is a contrastive focus which does not contribute information on the quantification domain of only. In other words, although the intended meaning of the but-clause is of all the subjects that I like, the only subject I like is semantics, the given prosodic focus on $L I K E$ fails to establish the appropriate set of alternatives namely: all the subjects that I like. Such cases clearly involve inference, possibly a reasoning along the following lines: the but conjunction indicates an expectation denial. The expectation is that if $x$ takes maths and semantics then $x$ likes maths and semantics. This expectation is thus made salient by the discourse context and provides in fact the set of alternatives necessary to interpret only namely the set $\{$ like (i,sem), like(i,maths)\}. To be more specific, consider the representation of $I$ only like semantics:

$$
\forall P[P \in F V \wedge P \rightarrow P=\operatorname{like}(i, \operatorname{sem})]
$$

By resolving $F V$ to the set of propositions $\{$ like(i,sem), like(i,maths)\}, we get the appropriate meaning namely:

$$
\begin{aligned}
& \forall P[P \in\{\operatorname{like}(i, \text { sem }), \operatorname{like}(i, \text { maths })\} \wedge P \\
& \rightarrow P=\operatorname{like}(i, \text { sem })]
\end{aligned}
$$

Following (Rooth, 1992b), I assume that in such cases, the quantification domain of both usually and only are pragmatically determined. 
The redundancy constraint on deaccenting still holds but it plays no role in determining these particular quantification domains.

\section{Sloppy identity}

As we saw in section 2, an important property of DSP's analysis is that it predicts sloppy/strict ambiguity for VP-Ellipsis whereby the multiple solutions generated by HOU capture the multiple readings allowed by natural language. As (Hobbs and Kehler, 1997; Hardt, 1996) have shown however, sloppy identity is not necessarily linked to VP-ellipsis. Essentially, it can occur whenever, in a parallel configuration, the antecedent of an anaphor/ellipsis itself contains an anaphor/ellipsis whose antecedent is a parallel element. Here are some examples.

Jon ${ }^{1}$ [took his 1 wife to the station f $^{2}$. No, BILL [took his wife to the station] 2 . (Bill took Bill's wife to the station)

Jon $^{1}$ spent [his s $_{1}$ paycheck $]^{2}$ but Peter saved it ${ }_{2}$. (Peter saved Peter's paycheck)

I'll [help you] $]^{1}$ if you [want me to $]^{2}$. I'll kiss you if you don't $t_{2}$. (I'll kiss you if you don't want me to kiss you)

Because the HOU-analysis reconstructs the semantics common to source and target rather than (solely) the semantics of VP-ellipses, it can capture the full range of sloppy/strict ambiguity illustrated above (and as (Gardent, 1997) shows some of the additional examples listed in (Hobbs and Kehler, 1997)). Consider for instance example (16). The ellipsis in the target has an antecedent want me to which itself contains a VPE whose antecedent (help you) has a parallel counterpart in the target. As a result, the target ellipsis has a sloppy interpretation as well as a strict one: it can either denote the same property as its antecedent VP want me to help you, or its sloppy copy namely want me to kiss you.

The point to note is that in this case, sloppy interpretation results from a parallelism between VPs not as is more usual, from a parallelism between NPs. This poses no particular problem for the HOU-analysis. As usual, the parallel elements (help and kiss) determine the equational constraints so that we have the fol- lowing equalities ${ }^{7}$ :

$$
\begin{aligned}
& C(h)=w t(\text { you }, h(i, \text { you })) \rightarrow h(i, \text { you }) \\
& C(k)=P(\text { you }) \rightarrow k(i, \text { you })
\end{aligned}
$$

Resolution of the first equation yields $\lambda R . w t(y o u, R(i, y o u)) \rightarrow R(i$, you $)$ as a possible value for $C$ and consequently, the value for $C(k)$ is:

$$
C(k)=w t(\text { you }, k(i, \text { you })) \rightarrow k(i, \text { you })
$$

Therefore a possible substitution for $P$ is:

$$
\{P \leftarrow x \cdot w t(x, k(i, x))\}
$$

and the VPE occurring in the target can indeed be assigned the sloppy interpretation $x$ want me to kiss $x$.

Now consider example (15). The pronoun it occurring in the second clause has a sloppy interpretation in that it can be interpreted as meaning Peter's paycheck, rather than Jon's paycheck. In the literature such pronouns are known as paycheck pronouns and are treated as introducing a definite whose restriction is pragmatically given (cf. e.g. (Cooper, 1979)). We can capture this intuition by assigning paycheck pronouns the following representation:

$$
\text { Pro } \begin{aligned}
& \lambda Q . \exists x[P(x) \wedge \forall y[P(y) \\
& \rightarrow y=x] \wedge Q(x)]
\end{aligned}
$$

with $P \in w f f_{(e \rightarrow t)}$. That is, paycheck pronouns are treated as definites whose restriction $(P)$ is a variable of type $(e \rightarrow t)$. Under this assumption, (15) is assigned the following equations ${ }^{8}$ :

$$
\begin{aligned}
& C(j, s p)=\exists_{1} x\left[p c_{-} o f(x, j) \wedge s p(j, x)\right] \\
& C(p, s a)=\exists_{1} x[P(x) \wedge s a(p, x)]
\end{aligned}
$$

Resolving the first equation yields

$$
\lambda y \cdot \lambda O . \exists_{1} x\left[p c \_o f(x, y) \wedge O(y, x)\right]
$$

as a value for $C$, and therefore we have that:

$$
\begin{aligned}
& C(p, s a)=\exists_{1} x\left[p c_{-} o f(x, p) \wedge s a(p, x)\right] \\
& \left\{P \leftarrow \lambda y . p c_{-} o f(y, p)\right\}
\end{aligned}
$$

That is, the target clause is correctly assigned the sloppy interpretation: Peter saved Peter's paycheck.

\footnotetext{
${ }^{7}$ For simplicity, I've ommitted polarity information.

${ }^{8} \mathrm{I}$ abbreviate $\lambda Q . \exists x[P(x) \wedge \forall y[P(y) \rightarrow y=x] \wedge Q(x)]$ to $\lambda Q \cdot \exists_{1} x[P(x) \wedge Q(x)]$.
} 
Thus the HOU-treatment of parallelism can account for both paycheck pronouns and examples such as (16). Though lack of space prevents showing how the other cases of sloppy identity are handled, the general point should be clear: because the HOU-approach associates sloppy identity with parallelism rather than with VPellipsis, it can capture a fairly wide range of data providing some reasonable assumptions are made about the representations of ellipses and anaphors.

\section{Implementation}

It is known that for the typed lambda-calculus, HOU is only semi-decidable so that the unification algorithm need not terminate for unsolvable problems. Fortunately, the class of equations that is needed for semantic construction is a very restricted class for which much better results hold. In particular, the fact that free variables only occur on the left hand side of our equations reduces the problem of finding solutions to higher-order matching, a problem which is decidable for the subclass of thirdorder formulae (Dowek, 1992).

These theoretical considerations have been put into practice in the research prototype CHOLI, a system which permits testing the HOU-approach to semantic construction. Briefly, the system can: parse a sequence of sentences and return its semantic representation, interactively build the relevant equations (parallel elements are entered by the user and the corresponding equations are computed by the system) and solve them by means of HOU.

The test-suite includes approximately one hundred examples and covers the following phenomena:

- VP-ellipsis and its interaction with anaphora, proper nouns (e.g., Mary, Paul) and control verbs (i.e., verbs such as try whose subject "control" i.e., is co-referential with some other element in the verb complement).

- Deaccenting and its interaction with anaphora, VP-ellipsis, context and sloppy/strict ambiguity.

- Focus with varying and ambiguous foci. It is currently being extended to sentences with multiple foci and the interaction with deaccenting.

As mentioned in section 2 the HOU-approach sometimes over-generates and yields solutions which are linguistically invalid. However as (Gardent et al., 1999) shows, this shortcoming can be remedied using Higher-Order Colored Unification (HOCU) rather than straight HOU. In CHOLI both an HOU and an HOCU algorithm can be used and all examples have been tested with and without colors. In all cases, colors cuts down the number of generated readings to exactly these readings which are linguistically acceptable.

\section{Conclusion}

It should by now be clear that the DSPtreatment of ellipsis is better seen as a treatment of the effect of semantic parallelism: the equations constrain the interpretation of parallel structures and as a side effect, a number of linguistic phenomena are predicted e.g. VPEresolution, sloppy/strict ambiguity and focus value inheritance in the case of SOEs.

There are a number of proposals (Hobbs and Kehler, 1997; Prüst et al., 1994; Asher, 1993; Asher et al., 1997) adopting a similar approach to parallelism and semantics of which the most worked out is undoubtly (Hobbs and Kehler, 1997). (Hobbs and Kehler, 1997) presents a general theory of parallelism and shows that it provides both a fine-grained analysis of the interaction between VP-ellipsis and pronominal anaphora and a general account of sloppy identity. The approach is couched in the "interpretation as abduction framework" and consists in proving by abduction that two properties (i.e. sentence or clause meaning) are similar. Because it interleaves a co-recursion on semantic structures with full inferencing (to prove similarity between semantic entities), Hobbs and Kehler's approach is more powerful than the HOU-approach which is based on a strictly syntactic operation (no semantic reasoning occurs). Furthermore, because it can represent coreferences explicitely, it achieves a better account of the interaction between VP-ellipsis and anaphora (in particular, it accounts for the infamous "missing reading puzzles" of ellipsis (Fiengo and May, 1994)).

On the other hand, the equational approach 
provided by the HOU-treatment of parallelism naturally supports the interaction of distinct phenomena. We have seen that it correctly captures the interaction of parallelism and focus. Further afield, (Niehren et al., 1997) shows that context unification supports a purely equational treatment of the interaction between ellipsis and quantification whereas (Shieber et al., 1996) presents a very extensive HOU-based treatment of the interaction between scope and ellipsis.

\section{Acknowledgments}

I wish to thank the ACL anonymous referrees for some valuable comments; and Stephan Thater, Ralf Debusman and Karsten Konrad for their implementation of CHOLI. The research presented in this paper was funded by the DFG in SFB-378, Project C2 (LISA).

\section{References}

Nicholas Asher. 1993. Reference to abstract objects in discourse. Kluwer, Dordrecht.

Nicholas Asher, Daniel Hardt, and Joan Busquets. 1997. Discourse parallelism, scope and ellipsis. In Proceedings of SALT'g7, Palo Alto.

Robin Cooper. 1979. The interpretation of pronouns. In F. Heny and H.S. Schnelle, editors, Syntax and Semantics, number 10, pages 6193.

Mary Dalrymple, Stuart Shieber, and Fernando Pereira. 1991. Ellipsis and higher-order unification. Linguistics \& Philosophy, 14:399-452.

Gilles Dowek. 1992. Third order matching is decidable. In Proceedings of the 7th Annual IEEE Symposium on Logic in Computer Science (LICS-7), pages 2-10. IEEE Computer Society Press.

Robert Fiengo and Robert May. 1994. Indices and Identity. MIT Press, Cambridge.

Claire Gardent and Michael Kohlhase. 1996a. Focus and higher-order unification. In Proceedings of COLING'96, Copenhagen.

Claire Gardent and Michael Kohlhase. 1996b. Higher-order coloured unification and natural language semantics. In Proceedings of $A C L$ '96, Santa Cruz.

Claire Gardent, Michael Kohlhase, and Karsten Konrad. 1999. Higher-order coloured unification: a linguistic application. Technique et Science Informatiques, 18(2):181-209.
Claire Gardent. 1997. Sloppy identity. In Christian Retoré, editor, Logical Aspects of Computational Linguistics, pages 188-207. Springer.

Claire Gardent. 1999. Deaccenting and higherorder unification. University of the Saarland. Submitted for publication.

Daniel Hardt. 1996. Dynamic interpretation of vp ellipsis. To appear in Linguistics and Philosophy.

J. Hobbs and A. Kehler. 1997. A theory of parallelism and the case of VP ellipsis. In Proceedings of $A C L$, Madrid.

Gérard P. Huet. 1976. Résolution d'Équations dans des Langages d'ordre $1,2, \ldots, w$. Thèse d'État, Université de Paris VII.

Ray S. Jackendoff. 1972. Semantic Interpretation in Generative Grammar. The MIT Press.

Manfred Krifka. 1992. A compositional semantics for multiple focus constructions. In Joachim Jacobs, editor, Informationsstruktur and Grammatik. Heidelberg. Sonderheft 4.

Manfred Krifka. 1995. Focus and/or context: A second look at second occurence expressions. Unpublished Ms. University of Texas, Austin, February.

Joachim Niehren, Manfred Pinkal, and Peter Ruhrberg. 1997. A uniform approach to underspecification and parallelism. In Proceedings of $A C L^{\prime} 97$, pages 410-417, Madrid, Spain.

H. Prüst, R. Scha, and M. van den Berg. 1994. Discourse grammar and verb phrase anaphora. Linguistics $\&$ Philosophy, 17:261327.

Steve Pulman. 1997. Higher order unification and the interpretation of focus. Linguistics \& Philosophy, 20:73-115.

Mats Rooth. 1992a. Ellipsis redundancy and reduction redundancy. In Steve Berman and Arild Hestvik, editors, Proceedings of the Stuttgart Ellipsis Workshop, University of Stuttgart.

Mats Rooth. 1992b. A theory of focus interpretation. Natural Language Semantics, pages 75-116.

Stuart Shieber, Fernando Pereira, and Mary Dalrymple. 1996. Interaction of scope and ellipsis. Linguistics 83 Philosophy, 19:527-552. 